EFOMP Policy Statement

\title{
EFOMP policy statement 16: The role and competences of medical physicists and medical physics experts under 2013/59/EURATOM
}

\author{
Carmel J. Caruana ${ }^{1}$, Virginia Tsapaki, John Damilakis, Marco Brambilla, \\ Guadalupe Martín Martín, Asen Dimov, Hilde Bosmans, Gillian Egan, Klaus Bacher, \\ Brendan McClean \\ EFOMP, United Kingdom
}

\section{A R T I C L E I N F O}

\section{Keywords:}

EFOMP

Medical physicist

Medical physics expert

Role development

Competence profile

European directive

\begin{abstract}
A B S T R A C T
On 5 December 2013 the European Council promulgated Directive 2013/59/EURATOM. This Directive is important for Medical Physicists and Medical Physics Experts as it puts the profession on solid foundations and describes it more comprehensively. Much commentary regarding the role and competences has been developed in the context of the European Commission project "European Guidelines on the Medical Physics Expert" published as Radiation Protection Report RP174. The guidelines elaborate on the role and responsibilities under 2013/59/EURATOM in terms of a mission statement and competence profile in the specialty areas of Medical Physics relating to medical radiological services, namely Diagnostic and Interventional Radiology, Radiation Oncology and Nuclear Medicine. The present policy statement summarises the provisions of Directive 2013/59/ EURATOM regarding the role and competences, reiterates the results of the European Guidelines on the Medical Physics Expert document relating to role and competences of the profession and provides additional commentary regarding further issues arising following the publication of the RP174 guidelines.
\end{abstract}

\section{Introduction}

On 5 December 2013 the European Council promulgated Directive 2013/59/EURATOM "laying down basic safety standards for protection against the dangers arising from exposure to ionising radiation" (henceforth referred to in this policy statement as the 'Directive') [1]. This directive is important for the Medical Physicist (L 13/3 (29) of [1]) and Medical Physics Expert (MPE, Article 4 (49) of [1]) - here collectively referred to as Medical Physics Professionals (MPP) as it puts the role of MPP on solid foundations. It describes the role and competences of MPP in medical radiological services, meaning services "pertaining to radiodiagnostic and radiotherapeutic procedures, and interventional radiology or other medical uses of ionising radiation for planning, guiding and verification purposes" (Article 4(50)) and non-medical imaging exposure meaning "any deliberate exposure of humans for imaging purposes where the primary intention of the exposure is not to bring a health benefit to the individual being exposed" (Article 4(55)) much more comprehensively. In the case of 97/43/EURATOM [2] the role description was not prescriptive enough to provide sufficient guidelines for health care policy makers, service managers and indeed MPP.
The present policy statement provides elaboration, explanation and comment regarding the provisions of the Directive relating to the role and competences (responsibilities) of MPP. Much commentary has been developed in the context of the European Commission project "European Guidelines on the Medical Physics Expert", and published as Radiation Protection Report RP174 [3]. This important document will be referred to in this policy statement as the 'Guidelines'. Among other items, the Guidelines define the role and competences of MPP under 2013/59/EURATOM in terms of a mission statement and detailed competence profile (i.e., list of competences, which in the context of the European Qualifications Framework (EQF) means list of responsibilities [4]) in the specialty areas of Medical Physics relating to medical radiological services, namely Diagnostic and Interventional Radiology, ${ }^{2}$ Radiation Oncology and Nuclear Medicine. Iit should be noted that there is unfortunately great confusion in the literature regarding the different definitions of the word 'competence' [5]; to avoid all ambiguity, the definition of the European Commission as stated in the European Qualifications Framework [4] will be used throughout this policy statement). This policy statement also provides additional commentary regarding further issues arising following the publications of the Guidelines.

\footnotetext{
${ }^{1}$ Lead author of the role chapter and competence inventories in the 'European Guidelines on the Medical Physics Expert' document.

${ }^{2}$ Including interventions outside the Radiology department proper (e.g., cardiology, orthopaedics) and dental, oral and maxillofacial surgery.
} 


\section{Main articles of $2013 / 59 /$ EURATOM highlighting the role and competences of MPP}

The main articles of 2013/59/EURATOM giving prominence to the role and competences of MPP are the following:

\subsection{Article 4(49): definition of the medical physics expert}

"medical physics expert" means an individual or, if provided for in national legislation, a group of individuals, ${ }^{3}$ having the knowledge, training and experience to act or give advice on matters relating to radiation physics applied to medical exposure, whose competence in this respect is recognised by the competent authority.

\subsection{Article 83: medical physics expert}

1. Member States shall require the medical physics expert to act or give specialist advice, as appropriate, on matters relating to radiation physics for implementing the requirements set out in Chapter VII ('Medical Exposures') 4 and in point (c) of Article 22(4) ('Practices involving the deliberate exposure of humans for non-medical imaging purposes ") 5 of this Directive.

2. Member States shall ensure that depending on the medical radiological practice, the medical physics expert takes responsibility for dosimetry, including physical measurements for evaluation of the dose delivered to the patient and other individuals subject to medical exposure, give advice on medical radiological equipment, and contribute in particular to the following:

(a) optimisation of the radiation protection of patients and other individuals subject to medical exposure, including the application and use of diagnostic reference levels;

(b) the definition and performance of quality assurance of the medical radiological equipment;

(c) acceptance testing of medical radiological equipment;

(d) the preparation of technical specifications for medical radiological equipment and installation design;

(e) the surveillance of the medical radiological installations;

(f) the analysis of events involving, or potentially involving, accidental or unintended medical exposures;

(g) the selection of equipment required to perform radiation protection measurements;

(h) the training of practitioners and other staff in relevant aspects of radiation protection;

3. The medical physics expert shall, where appropriate, liaise with the radiation protection expert.

\subsection{Article 57: responsibilities}

1. Member States shall ensure that:

(b) the practitioner, the medical physics expert and those entitled to carry out practical aspects of medical radiological procedures ${ }^{6}$ are

\footnotetext{
3 "By 'group of individuals' is meant a group of MPP with at least one who has reached the status of MPE in each specialised area of radiation physics applied to medical exposure e.g., Diagnostic and Interventional Radiology or Radiation Oncology or Nuclear Medicine including significant sub-specialities of these e.g., Brachytherapy, Nuclear Medicine therapy, Interventional Imaging in Cardiology as owing to the rapid expansion in medical technology it is becoming increasingly difficult for any single individual to be able to act or give advice in all areas of radiation physics applied to medical exposure." This group of individuals together with any ancillary staff would form an independent department of Medical Physics Services.

${ }^{4}$ Chapter VII on Medical Exposures is very extensive and must be studied in depth by MPP as it is full of opportunities for the role to assert itself.

${ }^{5}$ This refers to a new category of practices that make use of medical imaging devices for non-medical purposes defined in the Directive.

6 "those entitled to carry out practical aspects of medical radiological procedures": such professionals may be Medical Physicists not yet at expert level, radiographers, dental nurses and others.
}

involved, as specified by Member States, in the optimisation process;

\subsection{Article 58: procedures}

Member States shall ensure that:

(d) in medical radiological practices, a medical physics expert is appropriately involved, the level of involvement being commensurate with the radiological risk posed by the practice. In particular:

(i) in radiotherapeutic practices other than standardised therapeutic nuclear medicine practices, a medical physics expert shall be closely involved;

(ii) in standardised therapeutical nuclear medicine practices as well as in radiodiagnostic and interventional radiology practices, involving high doses as referred to in point (c) of Article 61(1), a medical physics expert shall be involved;

(iii) for other medical radiological practices not covered by points (a) and (b), a medical physics expert shall be involved, as appropriate, for consultation and advice on matters relating to radiation protection concerning medical exposure.

\subsection{Article 22: practices involving the deliberate exposure of humans for} non-medical imaging purposes

1. Member States shall ensure the identification of practices involving non-medical imaging exposure, in particular taking into account the practices included in Annex V.

4. Where a Member State has determined that a particular practice involving non-medical imaging exposure is justified, it shall ensure that:

(c) for procedures using medical radiological equipment

(i) relevant requirements identified for medical exposure as set out in Chapter VII are applied, including those for equipment, optimisation, responsibilities, training and special protection during pregnancy and the appropriate involvement of the medical physics expert;

(ii) where appropriate, specific protocols, consistent with the objective of the exposure and required image quality, are put in place;

(iii) where practicable, specific diagnostic reference levels are put in place;

\section{Main elements of the guidelines highlighting the role and competences of MPP}

\subsection{Mission statement for MPP}

The mission statement for Medical Physicists and Medical Physics Experts based on 2013/59/EURATOM follows: "Medical Physicists and Medical Physics Experts will contribute to maintaining and improving the quality, safety and cost-effectiveness of healthcare services through patient-oriented activities requiring expert action, involvement or advice regarding the specification, selection, acceptance testing, commissioning, quality assurance/control and optimised clinical use of medical radiological devices and regarding patient risks from ionising radiations including protection from such radiations, installation design and surveillance, and the prevention of unintended or accidental exposures; all activities will be based on current best evidence or own scientific research when the available evidence is not sufficient. The scope includes risks to volunteers in biomedical research and carers and comforters" [3]. The purpose for the formulation of this mission statement was to summarise the role, make it more understandable to policy makers, regulatory authorities and the management of healthcare organizations and provide guidance to MPP themselves. 


\subsection{Competence profile of MPP}

In the European Qualifications Framework for Lifelong Learning [4] a 'competence' is defined as a responsibility. A competence profile is the list of competences (responsibilities) expected of a class of professionals. The complete inventory of the competences expected of all MPP and specific competences expected in each of the three specialities of medical physics addressed in this policy statement is too extensive to be reproduced here but can be found detailed in the Guidelines (Annex I). All MPP are asked to study this competence profile in detail and apply it to their practice. In the Guidelines the competences and the corresponding knowledge and skills are categorized as follows:

(a) Medical physics core competences expected of all MPP irrespective of their specialty: these are categorized as competences expected of MPP as physical scientists, those expected of MPP as healthcare professionals and those expected of MPP as experts in the clinical use of medical radiological devices and protection from associated ionising radiations.

(b) Medical physics competences specific for each medical physics specialty: these competences are highly specific to each specialty of medical physics. It is important to note that an MPP from one specialty of medical physics who is required to assume additional specific competences from another specialty should be certified to carry out those additional responsibilities. Such cases may arise for example in a small nuclear medicine facility which requires its nuclear medicine MPP to take responsibility for the management of quality control testing of the CT component of a PET/CT system or at a small radiation oncology facility which requires its radiation oncology MPE to take responsibility for protocol optimization of a planning CT.

The full list of competences can be found in the Guidelines. For convenience the competences are categorized under the Key Activity Areas of MPP listed below and defined in detail in Table 1.

(a) Scientific problem solving service;

(b) Dosimetry measurements;

(c) Patient safety/risk management (including volunteers in biomedical research, carers, comforters and persons subjected to nonmedical procedures using medical devices);

(d) Occupational and public safety/risk management when there is an impact on medical exposure or own safety;

(e) Clinical medical device management;

(f) Clinical involvement;

(g) Development of service quality and cost-effectiveness;

(h) Expert consultancy;

(i) Education of healthcare professionals (including medical physics trainees);

(j) Health technology assessment;

(k) Innovation.

\section{Commentary}

The Directive makes it clear that the role of MPP in hospitals is to take responsibility for dosimetry (measurements and calculations) of patients and other individuals subject to medical exposure. In addition, they are to use their comprehensive and in-depth physical and mathematical knowledge to ensure that medical radiological equipment (including radiation sources and software devices) in hospital is judiciously chosen according to the desired clinical outcomes, properly calibrated, quality controlled and very importantly used effectively, safely and efficiently for the good of the patient. They are to know the radiological equipment very well, study user and technical manuals in detail, keep abreast of latest International and European recommendations, standards, documentation and research findings, keep themselves updated on new technologies and software updates, and liaise in an ongoing manner with the radiological equipment industry and other health care professionals. MPP bring a scientific approach to the technological aspects of clinical service development involving radiological equipment which is unique. In particular they should ensure that:

(a) Hospitals buy those radiological devices (including radiation sources and software devices) having the technical specifications which would deliver the target clinical patient outcomes at acceptable or, for therapeutic purposes, required patient doses (specification and selection of medical devices) Article 83 (2)(d);

(b) Radiological equipment delivered to the hospital conforms to the tendered specifications and installed appropriately and safely with respect to patients, volunteers in biomedical research, carers and comforters and persons subjected to non-medical imaging procedures and in liaison with the Radiation Protection Expert safely with respect to staff and the general public (acceptance testing and installation design) Article 83 (2)(c)(d) and Article 83(3); It is to be noted that often the role of the Radiation Protection Expert is held also by MPP. This is proactively encouraged by EFOMP in it's Malaga Declaration [6] particularly since there are many instances when doses to patients and staff or carers are highly correlated e.g., interventional procedures, nuclear medicine. Article 19(4) states specifically that "Practices involving medical exposure shall be justified both as a class or type of practice, taking into account medical and, where relevant, associated occupational and public exposures, and at the level of each individual medical exposure as specified in Article 55".

(c) Radiological equipment settings are optimised to the health care needs and characteristics of the local patient population and patient groups, quality control benchmarks are established based on locally available quality control equipment and patient dose values established (commissioning ${ }^{7}$ ) Article 83 (2)(a);

(d) Radiological equipment is quality controlled on an ongoing basis and appropriate quality control protocols set-up (management and supervision of device quality control procedures; calibration and verification of measurement instruments; establishment of criteria for acceptable performance; carrying out any quality control procedures involving advanced physics, mathematics and computing) Article 83 (2)(b);

(e) Written use protocols for every type of standard medical radiological procedure and equipment (as required by Article 58(a)) ${ }^{8}$ are optimised in terms of clinical effectiveness, efficiency and reduction of risk to individual patients, specific patient groups, volunteers in biomedical research, carers and comforters. This may include analysis of patient data when this involves advanced physics, mathematics and computing. It also includes liaison with the Radiation Protection Expert to ensure radiation protection of staff and public. Article 83 (2)(a) and Article 83(3);

(f) Risks are managed well, unintended or accidental exposures prevented, unintended or accidental exposures (e.g., foetal doses) estimated and recommendations put forward to avoid future repeats. The scope includes risks incurred by carers and comforters and by volunteers in medical or biomedical research. Article $83(2)(\mathrm{e})(\mathrm{f})$ and Article $4(48)^{9} \cdot{ }^{10}$

\footnotetext{
${ }^{7}$ The Directive unfortunately does not distinguish between acceptance testing and commissioning, but does assume some aspects of commissioning within the acceptance testing itself.

${ }^{8}$ Article 58(a): "Written protocols for every type of standard medical radiological procedure are established for each equipment for relevant categories of patients".

${ }^{9}$ Article 4(48) "medical exposure means exposure incurred by patients or asymptomatic individuals as part of their own medical or dental diagnosis or treatment, and intended to benefit their health, as well as exposure incurred by carers and comforters and by volunteers in medical or biomedical research".

${ }^{10}$ This would include also the setting up of dose constraints (Article 6(1)(c) "for medical exposure, dose constraints shall apply only with regard to the protection of carers
} 
Table 1

The key activities of the medical physicist and medical physics expert.

Key Activity
Scientific problem solving service
Dosimetry measurements (all physical agents e.g., effective dose in ionising
radiation, SAR in MRI, thermal and mechanical indices in ultrasound)

radiation, SAR in MRI, thermal and mechanical indices in ultrasound)

Patient safety/risk management (including volunteers in biomedical research, carers, comforters and persons subjected to non-medical procedures using medical devices)

Occupational and public safety/risk management when there is an impact on medical exposure or own safety ${ }^{1}$

Clinical medical device management

Clinical involvement

Development of service quality and cost-effectiveness

Expert consultancy

Education of healthcare professionals (including medical physics trainees)

Health technology assessment (HTA)

Innovation
Main Actions

Comprehensive scientific problem solving service involving recognition of less than optimal performance or optimised use of medical devices, identification and elimination of possible causes or misuse, and confirmation that proposed solutions have restored device performance and use to acceptable status. All activities are to be based on current best scientific evidence or own research when the available evidence is not sufficient

Measurement and calculations of dose received by patients, volunteers in biomedical research, carers, comforters and persons subjected to non-medical procedures using medical devices for the purpose of supporting justification and optimization processes; selection, calibration and maintenance of dosimetry related instrumentation; independent checking of dose provided by dose reporting devices (including software devices); measurement of quantities required as inputs to dose reporting or estimating devices (including software). Measurements to be based on current recommended techniques and protocols

Surveillance of medical devices and evaluation of clinical protocols to ensure the on-going protection of patients, volunteers in biomedical research, carers, comforters and persons subjected to non-medical procedures using medical devices from the deleterious effects of physical agents in accordance with the latest published evidence or own research when the available evidence is not sufficient. Includes optimization, the development of risk assessment protocols, including the analysis of events involving, or potentially involving, accidental or unintended medical exposures to physical agents and dose audit

Surveillance of medical devices and evaluation of clinical protocols with respect to the protection of workers and public from physical agents when impacting the exposure of patients, volunteers in biomedical research, carers, comforters and persons subjected to non-medical procedures or responsibility with respect to own safety. Correlation of occupational, public and medical exposures - balancing occupational and public risk and patient needs

Provide technical advice and participate in the specification, selection, acceptance testing, commissioning, installation design and decommissioning of medical devices in accordance with the latest published European or International recommendations. The specification, management and supervision of associated quality assurance/control programmes. Design of all testing protocols is to be based on current European or international recommended techniques and protocols

Carrying out, participating in and supervising everyday patient physical agent protection and quality control procedures to ensure on-going effective and optimised use of medical devices and including patient specific optimization, prevention of unintended or accidental exposures and patient follow-up. Optimization of protocols before first use with patients via the use of anthropomorphic phantoms and simulation using specialised physical agent dosimetry software

Support the introduction of new medical devices into clinical service, lead the introduction of new medical physics services and participate in the introduction/development of clinical protocols/techniques whilst giving due attention to economic issues

Provision of expert advice to outside clients (e.g., smaller clinics with no in-house medical physics expertise)

Contributing to quality healthcare professional education through knowledge transfer activities concerning the technical-scientific knowledge, skills and competences supporting the clinically-effective, safe, evidence-based and economical use of medical devices. Participation in the education of medical physics students and organisation of medical physics residency programmes

Taking responsibility for the physics component of health technology assessments related to medical devices and/or the medical uses of physical agents

Contribution to scientific and technical advancement in medical physics, by developing new or modifying existing technologies, devices, practices, software, techniques, models, protocols, for improved solutions to diagnosis and treatment of disease

\footnotetext{
${ }^{1}$ When the reduction of occupational and public risk would have an impact on medical effectiveness and exposure to physical agents (e.g., in interventional radiology in which patient and occupational exposure are correlated, or nuclear medicine in which patient, occupational and public risk are correlated) optimization would require input from both an MPE and other experts e.g., Radiation Protection Expert (or an MPE recognized also as RPE). The MP and MPE are always required to have knowledge and skills in occupational radiation protection sufficient to take responsibility for own protection.
}

(g) Patient risk indicators are measured accurately and monitored (external and internal dosimetry and dose monitoring including the establishment of DRL). Article 83 (2) and Article 83 (2)(a)(g);

(h) Surveillance of medical radiological installations is carried out in an ongoing manner and unsafe devices are decommissioned. Article 83

(footnote continued)

and comforters and volunteers participating in medical or biomedical research".) (2)(e);

(i) All healthcare professionals are well trained with regard to technical aspects of optimised radiological medical device use and radiation protection. Article $83(2)(\mathrm{h})$.

The above tasks are summarised in the mission statement from the Guidelines. In addition the mission statement goes a step further and states the competence level at which these functions need to be exercised 
particularly at the MPE level i.e., "all activities will be based on current best evidence or own scientific research when the available evidence is not sufficient". This is an important statement which indicates that MPP must keep themselves updated on technological developments, international and European documentation, manufacturer documentation and the research literature with regard to radiological medical devices (including radiation sources and software devices), their use and radiation protection to be in a position to contribute dynamically to service and protocol development and quality. It is important to work with the other members of the healthcare team as the contribution of all is required to ensure an effective, safe and efficient service. The MPE should evaluate patient dose reports from representative samples of each procedure and patient group and discuss the results with device users taking into consideration the clinical effectiveness of the procedures and assisting in any necessary follow-up. This would in turn facilitate compliance with policies and regulations.

Article 58(d) requires that an MPE "is appropriately involved, the level of involvement being commensurate with the radiological risk posed by the practice". In the case of "radiotherapeutic practices other than standardised therapeutic nuclear medicine practices, a medical physics expert shall be closely involved" This means that in order to avoid acute cases of insufficient dose-coverage of target volumes or excessive damage to healthy tissues, an MPE must be present at all times during the entire patient pathway. In the case of external beam therapy or brachytherapy this means that the MPE is responsible for dosimetry from patient imaging for planning to patient discharge. Moreover, during times when patients are being irradiated the MPE should manage the monitoring of the therapeutic devices and the quality assurance/control and verification procedures in an ongoing manner. The MPE will take responsibility for treatment planning and ensure that for each patient "exposures of target volumes shall be individually planned and their delivery appropriately verified taking into account that doses to nontarget volumes and tissues shall be as low as reasonably achievable and consistent with the intended radiotherapeutic purpose of the exposure" (Article 56(1)). The MPE must supervise every dosimetric plan in order to achieve the treatment plan objectives, that is, to deliver the therapeutic dose to the planning target volume with the highest homogeneity and to keep the dose to the organs at risk as low as possible. For special techniques which include high dose gradients (IMRT, SBRT, SRS, VMAT, etc.), the MPE must perform or supervise in addition the dosimetric measurements required to ensure that calculated planned doses agree with the criteria of acceptability of the specific technique. The MPE must perform or supervise the independent checks of dose when appropriate. The MPE must take responsibility for understanding, commissioning and quality control of the treatment planning system as well as its output.

In the case of 'non-standardised therapeutic nuclear medicine practices', the MPE is responsible for quantitative functional imaging, quality assurance/control and individualized treatment planning with tracer diagnostics and verification of the delivered doses, wherever practically achievable and based on current best practice and evidence $[7,8]$.

The term 'standardised therapeutic nuclear medicine practice' was not defined in the directive. In this policy statement a 'standardised therapeutic nuclear medicine practice' and indeed any standardised radiological practice is defined as one which satisfies the following conditions (conversely a non-standardised practice is one where one or more of these conditions is not satisfied):

(a) It is in the form of a written protocol;

(b) It was developed by a multi-disciplinary team which included as a minimum a physician practitioner, an MPE in the specialty and a senior user;

(c) It's development is well-documented and based on international guidelines and current published best evidence (alternatively, when no international guidelines or published best evidence have been available, local research has been carried out to support the practice with the provision that the practice should be revised when international guidelines or published best practice become available);

(d) It has been updated at appropriate intervals;

(e) It is not currently under modification;

(f) It is used in situations with mostly predictable outcome in both efficacy and (low risk of) toxicity, and patient quality-of-life;

(g) The protocol includes a list of specific circumstances under which the physician practitioner and/or MPE should be contacted for action and/or advice.

(h) It involves the use of a registered radiopharmaceutical within its authorised clinical application.

Verification of the absorbed doses delivered to the target volumes and organs at risk are the minimum actions under responsibility of the MPE to ensure appropriate dose delivery by nuclear medicine therapy. The ALARA principle should be followed for nuclear medicine therapy to avoid unnecessary exposure of the patient, relatives and members of the public.

In the case of standardised therapeutical nuclear medicine practices as well as in radiodiagnostic and interventional radiology practices, involving high doses as referred to in point (c) of Article 61(1)(c) (which specifically includes also interventional radiology and computed tomography) an MPE shall be involved. This means that an MPE should monitor such practices regularly and be available on call at all times. These high dose practices together with paediatric and screening exposures are considered as 'special practices' (Article 61(1)), ${ }^{11}$ and should be given particular attention. Intensive ongoing dose monitoring (either through periodical clinical dose audit, individual patient dose calculations or dose monitoring software) should be applied in the case of screening as although the individual dose is low the population dose would be exceedingly high if protocols are not optimised by the MPE. The MPE shall prepare a specific QC and dose monitoring programme for each screening radiological system which should include typical acceptable patient doses, an estimate of the collective dose. Prior to first use a commissioning test shall be carried out by the MPE. In all imaging procedures the use of Diagnostic Reference Levels (DRL) as a first step in optimisation is crucial. The introduction of DRL for interventional radiology procedures is a new challenge required by the Directive. This task will require a significant effort for the MPE as monitoring these practices is difficult. Liaising with the RPE is essential to ensure adequate staff protection.

For other medical radiological practices not covered by the two previous risk categories, "a Medical Physics Expert shall be involved, as appropriate, for consultation and advice". This means that an MPE should be available on call at all times; however, a single MPE can be in charge of several small clinics on an outside consultancy basis. In both cases, it is imperative that the MPE (a) prepare a schedule of routine quality control tests to be carried by the radiological device users together with a form for assessment of routine image quality and patient dose (a users' technical report to be made available to the MPE on request) (b) prepare an appropriate schedule of visits to each radiological suite when the MPE would carry out more advanced evaluations of image quality and patient dose (c) set up written instructions indicating situations when the users are required to ask for advice and communicate this to management. The latter is particularly important as there have been instances when such users either did not realize that advice was necessary (as e.g., low image quality or high patient dose were not detected) or when advice was intentionally not

\footnotetext{
${ }^{11}$ Article 61 'Special Procedures'1. Member States shall ensure that appropriate medical radiological equipment, practical techniques and ancillary equipment is used in medical exposure: (a) of children; (b) as part of a health screening programme; (c) involving high doses to the patient, which may be the case in interventional radiology, nuclear medicine, computed tomography or radiotherapy. Special attention shall be given to quality assurance programmes and the assessment of dose or verification of administered activity for these practices.
} 
sought either as a result of budget pressures or excessive professional pride. Written instructions would ensure that advice is asked for in a timely manner and not when situations become critical or when it is too late to prevent unintended or accidental exposures or when image quality has deteriorated to a point that accurate diagnosis is compromised. Such written instructions will serve to protect MPP from any malpractice lawsuits resulting from the absence of timely requests for advice by users.

Article 22 ('Practices involving the deliberate exposure of humans for non-medical imaging purposes') refers to a new category of practices that make use of medical radiological devices for such procedures as: (a) radiological health assessment for employment purposes (b) radiological health assessment for immigration purposes (c) radiological health assessment for insurance purposes (d) radiological evaluation of the physical development of children and adolescents with a view to a career in sports, dancing, etc. (e) radiological age assessment (f) use of ionising radiation for the identification of concealed objects within the human body. (See Annex V of the Directive). Article 22(4)(c)(i) requires the involvement of the MPE in such practices including for optimisation and the establishment of DRLs and represents new opportunities for MPP.

The responsibility for justification of radiological procedures is that of the practitioner and referrer. However proper justification must be based on an evidence-based and quantitative evaluation of the benefits of and risks from the procedure. This is important for all procedures but particularly so in the case of paediatric exposures, screening, nonstandardised procedures, procedures involving risk to carers and comforters and medical and biomedical research. The MPE is required to provide the dosimetry to quantify risk in terms of appropriate dosimetric calculations/measurements. Such dose data should be kept by the licensee in accordance with the requirements of national legislation and advice provided by MPE.

The role of the MPP in non-ionising radiation as an alternative to ionising radiation in medical imaging is an issue which is not addressed in the Directive as the latter is dedicated solely to ionising radiation. However Article 55 regarding justification does state that: "Medical exposure shall show a sufficient net benefit, weighing the total potential diagnostic or therapeutic benefits it produces, including the direct benefits to health of an individual and the benefits to society, against the individual detriment that the exposure might cause, taking into account the efficacy, benefits and risks of available alternative techniques having the same objective but involving no or less exposure to ionising radiation". This ambiguity in the Directive renders the role of the MPP in giving advice regarding the relative effectiveness and safety of the various non-ionising imaging modalities difficult (although in practice giving such advice has become the norm) [9]. The Guidelines document also states that: "as the linking of non-ionising radiation devices to ionising radiation devices is on the increase (e.g., PET/MRI, SPECT/MRI, linac MRI, use of MRI and ultrasound in radiotherapy planning and monitoring), it is highly recommended that an MPE is appropriately knowledgeable regarding the medical use of such other physical agents $^{12}$ ". In fact the Guidelines document also lists knowledge and skills in MRI and ultrasound and although these are not mandatory, these learning outcomes have become the norm for MPE specialised in Diagnostic and Interventional Radiology.

\section{Role development}

The role and competences of every profession are not static. Each profession strategically seeks to expand and develop it's role by taking

\footnotetext{
12 'Physical Agents' is the legal term used by the European Commission for physics based sources of energy such as non-ionising radiations, optical sources, sound, vibrations etc. There are several directives involving protection from such sources particularly in the area of occupational safety. Although not referred to us such, ionising radiations and particle beams are of course also physical agents under this definition.
}

advantage of all political, legal, economic, social and technological opportunities available [10-12]. Directive 2013/59/EURATOM is an excellent legal opportunity for the profession to develop it's role further. Chapter VII of the Directive ('Medical Exposures') must be studied very intensively by MPP as it is full of opportunities for the profession to exercise and expand its role. Examples are Article 63 regarding accidental and unintended exposures ${ }^{13}$ and Article 64 on estimates of population doses from medical exposures. ${ }^{14}$ Other articles in other chapters also offer opportunities e.g., Chapter IX Article 96 on notification and recording of significant events. ${ }^{15}$ MPP are ideally placed to liaise with radiation protection authorities in such situations.

2013/59/EURATOM like every European directive is not revised often enough to keep abreast with developments in radiation protection documentation and research. Again directives are compromise documents that balance the interests of many stakeholders, and therefore are sometimes not sufficiently prescriptive for achieving state-of-the-art protection. Because of these reasons MPP should look beyond the directive for further guidance. Many organizations (e.g., IAEA, ICRP, EFOMP, AAPM) produce excellent documents which can be used by MPP to develop their role $[13,14]$. MPP are also very well-positioned to contribute to the ISO 9000 and ISO 17025 accreditation of medical organizations.

It is up to the National Member Organizations and the individual MPP to grasp these opportunities. EFOMP will help by providing necessary policy statements, supporting documentation and opportunities for advanced education and training but proactive strategic leadership by National Member Organizations at the national level is critical.

\section{Independent medical physics services}

Medical Physics Services are defined as individual MPP or groups of MPP who individually or collectively possess the knowledge, skills and experience to deliver effectively, safely and efficiently the complete set of competences expected of a Medical Physics Expert in all specialties of medicine and healthcare particularly those involving the use of ionising radiation. Such services can best be delivered by an independent Medical Physics department whose head reports directly at a very high to hospital management.

\footnotetext{
${ }^{13}$ Article 63: Accidental and unintended exposures Member States shall ensure that: (a) all reasonable measures are taken to minimise the probability and magnitude of accidental or unintended exposures of individuals subject to medical exposure; (b) for radiotherapeutic practices the quality assurance programme includes a study of the risk of accidental or unintended exposures; (c) for all medical exposures the undertaking implements an appropriate system for the record keeping and analysis of events involving or potentially involving accidental or unintended medical exposures, commensurate with the radiological risk posed by the practice; (d) arrangements are made to inform the referrer and the practitioner, and the patient, or their representative, about clinically significant unintended or accidental exposures and the results of the analysis; (e) (i) the undertaking declares as soon as possible to the competent authority the occurrence of significant events as defined by the competent authority; (ii) the results of the investigation and the corrective measures to avoid such events are reported to the competent authority within the time period specified by the Member State; (f) mechanisms are in place for the timely dissemination of information, relevant to radiation protection in medical exposure, regarding lessons learned from significant events.

${ }^{14}$ Article 64 Estimates of population doses "Member States shall ensure that the distribution of individual dose estimates from medical exposure for radiodiagnostic and interventional radiology purposes is determined, taking into consideration where appropriate the distribution by age and gender of the exposed".

${ }^{15}$ Article 96 Notification and recording of significant events "Member States shall require the undertaking to: (a) implement, as appropriate, a recording and analysis system of significant events involving or potentially involving accidental or unintended exposures; (b) promptly notify the competent authority of the occurrence of any significant event resulting or liable to result in the exposure of an individual beyond the operational limits or conditions of operation specified in authorising requirements with regard to occupational or public exposure or as defined by the competent authority for medical exposure, including the results of the investigation and the corrective measures to avoid such events".
} 


\section{Summary recommendations}

EFOMP strongly encourages its National Member Organizations to strive to ensure that the roles and responsibilities of MPP in their respective states comply with the recommendations in this policy statement. EFOMP also recommends that National Member Organizations produce a manual of good practice for their members in the national language and also conduct the necessary studies to determine the material resources and number of MPP at both entry and expert levels they would require in order to deliver the above services. The latter document should be delivered to the appropriate authorities; it will help safeguard staff of Medical Physics Services from any malpractice lawsuits arising from insufficient material and human resources.

\section{Acknowledgments}

The authors would like to acknowledge all EFOMP Officers, E. Vano, R. Padovani and delegates who have contributed to the final version of this document with comments and suggestions. In addition, EFOMP would like to thank the European Commission for providing the funds to make the European 'Guidelines on the Medical Physics Expert' project possible.

\section{References}

[1] EC. COUNCIL DIRECTIVE 2013/59/EURATOM of 5 December 2013 laying down basic safety standards for protection against the dangers arising from exposure to ionising radiation, and repealing Directives 89/618/Euratom, 90/641/Euratom, 96/29/Euratom, 97/43/Euratom and 2003/122/Euratom.

[2] EC. Directive 1997/43/Euratom of 30 June 1997 on health protection of individuals against the dangers of ionizing radiation in relation to medical exposure.
[3] EC. RP174 European Guidelines on the Medical Physics Expert; 2014.

[4] European Parliament and Council. Recommendation 2008/C 111/01 on the establishment of the European Qualifications Framework for lifelong learning.

[5] Castillo J, Caruana CJ, Wainwright D. The changing concept of competence and categorisation of learning outcomes in Europe: implications for the design of higher education radiography curricula at the European level. Radiography 2011;17(3):230-4.

[6] EFOMP's position on medical physics in Europe, 2006 ('Malaga Declaration').

[7] Chiesa C, Sjogreen Gleisner K, Flux G, Gear J, Walrand S, Bacher K, et al. The conflict between treatment optimization and registration of radiopharmaceuticals with fixed activity posology in oncological nuclear medicine therapy. Eur J Nucl Med Mol Imaging 2017;44:1783-6.

[8] Strigari L, Konijnenberg M, Chiesa C, Bardies M, Du Y, Sjögreen Gleisner K, et al, The evidence base for the use of internal dosimetry in the clinical practice of molecular radiotherapy. Eur J Nucl Med Mol Imaging 2014;41(10):1976-88.

[9] Hand J, Bosmans H, Caruana CJ, Keevil S, Norris DG, Padovani R, et al. EFOMP Policy Statement No 14: the role of the Medical Physicist in the management of safety within the magnetic resonance imaging environment: EFOMP recommendations. Phys Med 2013;29(2):122-5.

[10] Caruana CJ, Wasilewska-Radwanska M, Aurengo A, Dendy PP, Karenauskaite V, Malisan MR, et al. A strategic development model for the role of the biomedical physicist in the education of healthcare professionals in Europe. Phys Med 2012;28(4):307-18.

[11] Caruana CJ, Wasilewska-Radwanska M, Aurengo A, Dendy PP, Karenauskaite V, Malisan MR, et al. A comprehensive SWOT audit of the role of the biomedical physicist in the education of healthcare professionals in Europe. Phys Med 2010;26(2):98-110.

[12] Caruana CJ, Cunha JAM, Orton CG. Subjects such as strategic planning, extra-disciplinary communication, and management have become crucial to medical physics clinical practice and should become an integral part of the medical physics curriculum. Med Phys 2017;44:3885-7. http://dx.doi.org/10.1002/mp.12211.

[13] Delis H, Christaki K, Healy B, Loreti G, Poli GL, Toroi P, et al. Moving beyond quality control in diagnostic radiology and the role of the clinically qualified medical physicist. Phys Med 2017;41:104-8. http://dx.doi.org/10.1016/j.ejmp. 2017.04.007. Epub 2017 Apr 12.

[14] Berris T, Nüsslin F, Meghzifene A, Ansari A, Herrera-Reyes E, Dainiak N, et al. Nuclear and radiological emergencies: building capacity in medical physics to support response. Phys Med 2017;42:93-8. 